\title{
Locally advanced paranasal sinus carcinoma: A study of 30 patients
}

\author{
FRANCESCO PERRI $^{1}$, RAFFAELE ADDEO ${ }^{2}$, MANUEL CONSON $^{3}$, ADRIANA FAIELLA $^{3}$, \\ GIUSEPPINA DELLA VITTORIA SCARPATI ${ }^{1}$, GABRIELLA TORRE $^{3}$, ANGELA DI BIASE ${ }^{3}$, PAOLA ROMANELLI ${ }^{3}$, \\ CARLO BUONERBA $^{4}$, GIUSEPPE DI LORENZO ${ }^{5}$, ANTONIO DAPONTE ${ }^{6}$, FRANCESCO CAPONIGRO ${ }^{6}$, \\ SALVATORE PISCONTI $^{1}$, ROBERTO PACELLI ${ }^{2}$, VINCENZO RAVO $^{7}$, PAOLO MUTO $^{7}$ and RAFFAELE SOLLA ${ }^{8}$ \\ ${ }^{1}$ Medical Oncology Unit, 'Santissima Annunziata' Hospital, I-74100 Taranto; ${ }^{2}$ Oncology Unit, 'ASL Napoli 2 Nord', \\ San Giovanni di Dio Hospital, I-80027 Naples; ' Department of Radiation Therapy, University of Naples 'Federico II', \\ I-80131 Naples; ${ }^{4}$ Oncological Referral Center of Basilicata, The Institute for Research, Hospitalization and Health Care, \\ Rionero in Vulture, I-85028 Potenza; ${ }^{5}$ Department of Endocrinology and Molecular and Clinical Oncology, University of \\ Naples 'Federico II'; ${ }^{6} \mathrm{Head}$ and Neck Medical Oncology Unit, National Tumour Institute of Naples, The Institute for \\ Research, Hospitalization and Health Care 'Fondazione Giovanni Pascale'; ${ }^{7}$ Department of Radiotherapy, The Foundation \\ Institute for Research, Hospitalization and Health Care 'Istituto Nazionale dei Tumori', I-80131 Naples; \\ ${ }^{8}$ Institute of Biostructure and Bioimaging, National Council of Research, I-80128 Naples, Italy
}

Received November 4, 2015; Accepted May 23, 2016

DOI: $10.3892 / \mathrm{ol} .2017 .5598$

\begin{abstract}
Sinonasal carcinomas (SNcs) are rare neoplasms arising from the paranasal sinuses and nasal cavity. Although these tumours have a heterogeneous histology, they are commonly diagnosed as a locally advanced disease and are associated with a poor prognosis. The present retrospective study reviewed 30 patients with locally advanced SNc, who were treated with surgery followed by chemoradiotherapy or radiotherapy, or radiotherapy with or without concomitant chemotherapy between January 1999 and January 2013 at the Department of Radiation Therapy, University of Naples 'Federico II' (Naples, Italy). A total of 19 patients were treated with upfront surgery followed by adjuvant radio- or chemoradiotherapy (group A), while the remaining 11 patients received exclusive radiotherapy with or without concomitant chemotherapy (group B). Concurrent cisplatin-based chemotherapy (100 mg/m² days 1, 22 and 43 for 3 cycles) was administered to $34 \%$ of patients in group A and $55 \%$ of patients in group B. At a median follow-up of 31 months, $33.3 \%$ of patients were alive. Cause-specific survival (CSS) and progression-free survival (PFS) times were 32 and 12 months, respectively. No difference in CSS rate was observed between the two treatment groups. Univariate analysis determined that disease stage
\end{abstract}

Correspondence to: Dr Francesco Perri, Medical Oncology Unit, 'Santissima Annunziata' Hospital, Via per Martina Franca, I-74100 Taranto, Italy

E-mail: francesco.perri80@alice.it

Key words: paranasal sinus carcinoma, nasal cavity tumours, radiotherapy, locally advanced, three-dimensional conformal radiotherapy, chemotherapy was the only factor that significantly affected CSS $(\mathrm{P}=0.002)$ and PFS $(\mathrm{P}=0.0001)$ rates. Acute and chronic toxicities were mild, with only $23.3 \%$ of patients reporting G1-2 side effects and no treatment-related blindness. The present study reported moderate activity and efficacy of surgery followed by adjuvant radio- or chemoradiotherapy, and exclusive radiotherapy with or without chemotherapy in this poor prognosis category of patients.

\section{Introduction}

The sinonasal cavities are anatomical regions affected by a number of tumours that are clinically, genetically and etiologically different from classical carcinomas of the head and neck (1). Sinonasal carcinoma $(\mathrm{SNc})$ is a rare disease that accounts for $<3 \%$ of all head and neck tumours, with a 5-year overall survival (OS) rate of $30 \%$ across all stages. SNc is etiologically-associated with professional exposure to leather and wood dust particles, and is therefore defined as an occupational disease (2-4). Tumours of the maxillary sinuses are more prevalent than those of the nasal cavities and ethmoid sinuses (5). Squamous cell carcinoma and adenocarcinoma are the most frequent histological types, accounting for $80 \%$ of all SNcs, while neuroendocrine, adenoid cystic and undifferentiated entities are much less frequent (6).

Patients with SNcs are often asymptomatic in early stages and are therefore commonly diagnosed at an advanced stage (T3-4), presenting with a large primary tumour that invades the surrounding bone structures and is associated with a high frequency of poor outcome and local failure (7). Due to their rarity, there is a lack of randomized clinical trials assessing the standard treatment options for SNcs, with no clear guidelines concerning their treatment. Generally, surgery, whenever possible, represents the cornerstone of therapy in early (T1-2) 
and advanced stage (T3-4) patients, and should always be followed by adjuvant radiation therapy, except in cases of T1 low-risk disease (absence of involved surgical margins). Chemotherapy should be administered concomitantly with radiation therapy in cases of high-risk disease (T3-4 and/or N+ and/or involved surgical margins) (1,8-11). The outcome of patients with SNc also depends on histological type (12), and prognosis is poorer in patients with squamous cell carcinoma compared with adenocarcinoma (25-50 vs. 40-60\%, respectively) $(13,14)$. Undifferentiated sinonasal carcinoma (SNUc) often presents as a rapidly enlarging and decaying mass, which is associated with the poorest prognosis among all SNcs (15).

Adjuvant radiotherapy may offer an increased chance for local control, particularly in cases of high-risk disease; however, its utility must be balanced against its frequent optic nerve toxicity (16). Radiotherapy-induced blindness may occur in up to $40 \%$ of treated patients (17). Exclusive radiotherapy may be administered in cases of unresectable disease and should be coupled with chemotherapy, even if this does not represent the standard of care (18). Advanced disease is often treated with exclusive chemotherapy even if SNCs are poorly chemosensitive. The most effective drugs are the platinum-derived compounds, either associated with or not with 5-fluorouracil, and in certain cases they are administered with taxanes (19-22). At present, there are only a few clinical trials assessing the efficacy of targeted therapy in this category of tumours (23).

The present retrospective study mainly aimed to describe the survival of patients affected by this rare disease treated at University of Naples 'Federico II' (Naples, Italy). The current study also discusses the best therapy choice for locally advanced disease.

\section{Patients and methods}

A total of 30 patients with SNc treated between January 1999 and January 2013 at the Department of Radiation Therapy, University of Naples 'Federico II' were included in the present retrospective study. Pretreatment evaluation consisted of a complete patient history, physical examination and fine-needle aspiration with cytological examination to provide a diagnosis. A core-biopsy of the lesion was performed in all patients, which aimed to confirm initial diagnoses and histologically characterize lesions. Staging was completed with head and neck magnetic resonance imaging and thorax computed tomography (CT). All patients attended a multidisciplinary head and neck conference at the Department of Radiation Therapy, University of Naples 'Federico II'. Based on the National Comprehensive Cancer Network guidelines (24) and, primarily, the shared opinion of the multidisciplinary team, patients were selected for treatment with surgery followed by adjuvant radio- or chemoradiotherapy (group A), or definitive radiotherapy with or without concomitant chemotherapy (group B). Surgery aimed to achieve complete resection of the tumour with negative margins. The type and extension of the surgery was dictated by the onset site and the extent of the disease, in addition to functional considerations. The surgical techniques employed included total or subtotal maxillectomy and/or ethmoidectomy, and craniofacial resection. Lymph node resection was performed only in the presence of their clinical involvement. Criteria for unresectability included wide intradural or intracranial spread, encasement of the carotid artery and invasion of the cavernous sinus. Patients who were unsuitable for resection underwent definitive radiotherapy with or without concomitant chemotherapy. All patients were treated with three-dimensional-conformal radiotherapy. Treatment planning was based on CT examination performed with patients in a supine position using head-neck-shoulder thermoplastic devices. The target and organs at risk (OARs) were defined on a CT planning scan. Clinical target volume (CTV) included the macroscopic extent of disease/resection cavity/postoperative residual mass plus all paranasal sinuses that had been invaded or were at high risk of invasion. In case of orbital invasion, CTV included the medial region of the orbit. Elective nodal irradiation was not performed, but only the clinically positive lymph nodes were enclosed in the CTV. Planning target volume was defined as CTV plus a 5-mm isotropic margin. OARs included the retina, optic nerve and optic chiasm. A median total dose of $60 \mathrm{~Gy}$ (range, 50-64 Gy) in 30 fractions daily of $2 \mathrm{~Gy}$ was planned. The maximum dose of 54 Gy was used as dose constraint for OARs for planning elaboration. The fields were arranged and weighted to achieve the maximum possible uniform distribution in the target volume (95\% of prescription dose delivered to at least $95 \%$ of the PTV) without exceeding the dose constraints for the OARs. Concomitant cisplatin was administered at a dose of $100 \mathrm{mg} / \mathrm{m}^{2}$ on days 1,22 and 43 for 3 cycles during radiotherapy. This schedule was used for adjuvant and exclusive radiotherapy settings. Demographic, disease and treatment characteristics of all patients are presented in Table I.

Acute and chronic treatment-related toxicities were registered according to the Radiation Therapy Oncology Group/European Organization for Research and Treatment of Cancer toxicity scale (25).

Statistical analysis. The actuarial OS, cause-specific survival (CSS; percent of patients who succumbed to tumour progression) and progression-free survival (PFS) rates were estimated using the Kaplan-Meier method. Univariate analysis was performed using the log-rank test, aiming to investigate the effect of clinical and treatment-related variables on the 2-year CSS and PFS rates. All statistical tests were two-sided and $\mathrm{P}<0.05$ was considered to indicate a statistically significant difference. All statistical analyses were performed using SPSS 18.0 (SPSS, Inc., Chicago, IL, USA).

\section{Results}

A total of 19/30 patients were treated with upfront surgery followed by adjuvant radio- or chemoradiotherapy (group A), while the remaining 11 received exclusive radiotherapy with or without concomitant chemotherapy (group B). Concomitant chemotherapy was performed in 6/19 patients in group A, and in $6 / 11$ patients in group B. A total of $8 / 30$ patients were diagnosed as stage III [according to Tumour-Node-Metastasis staging (26)], while the remaining 22 were diagnosed as having stage IV disease. Histological types widely varied, with the majority determined as squamous cell carcinoma (Table I). At a median follow-up of 31 months (range, 6-148 months), 33.3\% of patients were alive and $90 \%$ of these did not experience 
Table I. Demographic, clinical and treatment characteristics of patients.

\begin{tabular}{ll}
\hline Characteristic & $\mathrm{n}(\%)$ \\
\hline
\end{tabular}

\section{Gender}

Female

$12(40.0)$

Male

$18(60.0)$

T onset site

Maxillary sinus

Nasal cavity

Ethmoidal sinus

Sphenoidal sinus

$2(6.7)$

Histological type

Squamous cell carcinoma

$16(53.3)$

Adenocarcinoma

$5(16.7)$

$5(16.7)$

$3(10.0)$

$1(3.3)$

Sinonasal undifferentiated carcinoma

Intestinal-like

III

$22(73.3)$

IV

T3

$\mathrm{T} 4$

$\mathrm{N}$

$\mathrm{N}-$
$\mathrm{N}+$

Grading

G1

G2

G3

Surgery

No

Yes

R0

R1

$11(36.7)$

$7(23.3)$

Chemotherapy

No

Yes

Neoadjuvant

Concentrated

Neoadjuvant + concentrated

$27(90.0)$

$3(10.0)$

$11(36.7)$

$10(33.3)$

$9(30.0)$

$12(40.0)$

$4(13.3)$

Patients ranged from 29-83 years of age with an average of 60 years.

relapse. Overall, the relapse rate was $76.7 \%$ (23/30), and the rate of local and distant recurrence was $82.6 \%(19 / 23)$ and 8.7\% (2/23), respectively. The estimated median CSS and PFS times were 32 and 12 months, respectively. Univariate analysis determined that only disease stage significantly affected the CSS $(\mathrm{P}=0.002)$ and PFS $(\mathrm{P}=0.0001)$ rates. The 2-year CSS rate was $87.5 \%$ for stage III and $54.5 \%$ for stage IV $(\mathrm{P}=0.016)$, and the 2-year PFS rate was $71.4 \%$ for stage III and $17.1 \%$ for
B

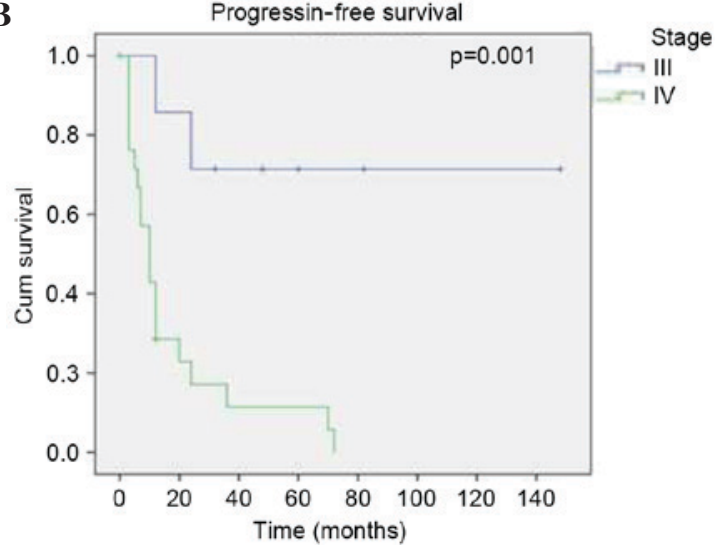

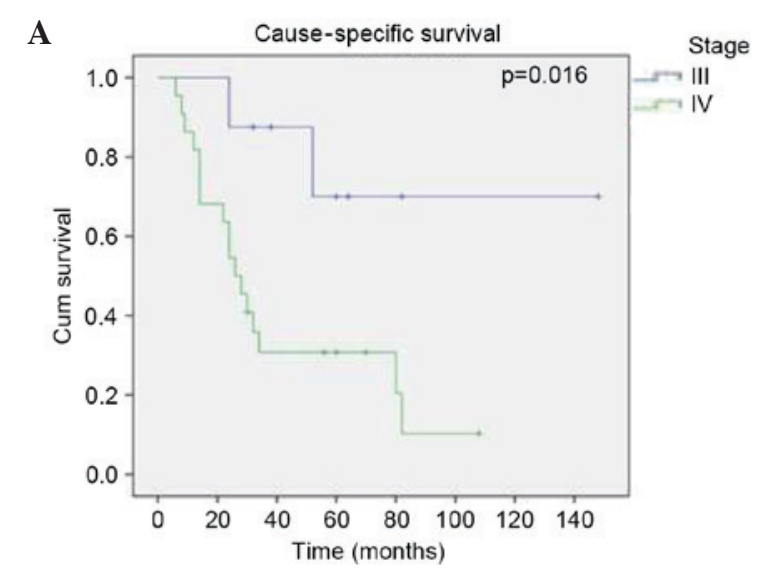

Figure 1. Kaplan-Meier estimated cumulative (A) cause-specific survival and (B) progression-free survival curves, stratified according to stage.

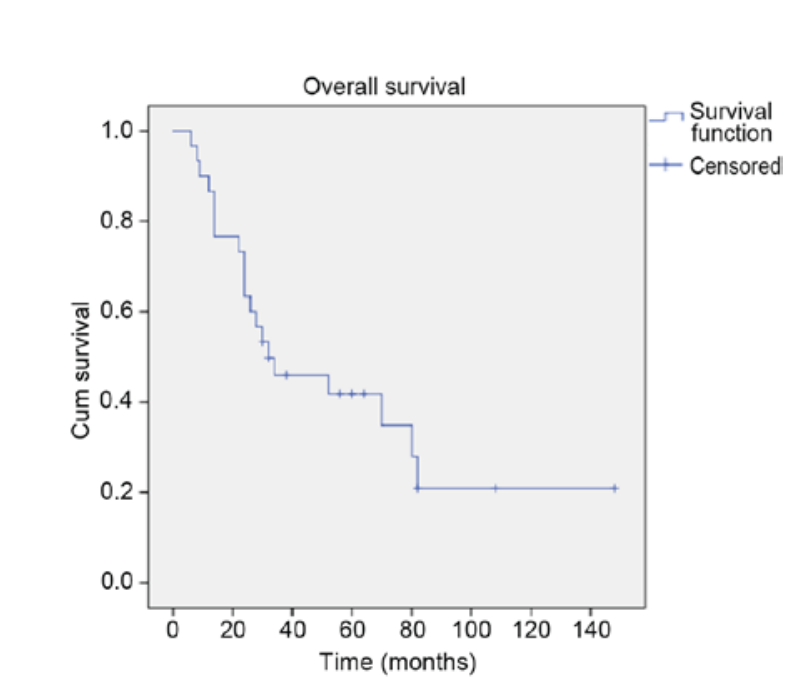

Figure 2. Kaplan-Meier estimated cumulative overall survival curve.

stage IV ( $\mathrm{P}=0.001)$ (Fig. 1). The effect of stage on CSS and PFS rates was essentially linked to the primary tumour size (T), and no contribution was observed for nodal status $(\mathrm{N})$; this was most likely due to the low number of lymph node-positive cases (3/30 patients). Notably, no significant differences in the 2-year CSS and PFS rates were observed between the surgery (group A) and no surgery (group B) subgroups. However, this result may be due to non-radical surgery, which was performed in $7 / 11$ resected patients. Non-radical surgery (R1 resection) is not as effective as radical surgery, being associated with lower 
survival in clinical trials. The constant presence of disease in the tumour bed is frequently associated with early local recurrence (27).

Similarly, no significant differences in CSS and PFS rates were observed between the chemotherapy and no chemotherapy subgroups in groups A and B. The results of the survival analysis are presented in Fig. 2. The remaining variables, including histological type, grade of differentiation and site of origin, did not significantly affect the CSS and PFS rates.

With regard to acute toxicity, $66.7 \%$ of patients (20/30) experienced side effects during radiation therapy. The most frequent side effects were oral mucositis and xerostomia, while other side effects included dysphagia and skin toxicity. These effects occurred at a median radiation dose of $30 \mathrm{~Gy}$, with a peak incidence at a median dose of $45 \mathrm{~Gy}$. With regard to acute toxicity grading, $43.3 \%$ (13/30) of patients developed grade 1 or grade 2 toxicity, while $23.3 \%$ of patients (7/30) developed grade 3 toxicity. Chronic side effects were observed in $13.3 \%$ of patients (4/30), represented by the presence of xerostomia in all 4 cases. No events of chronic toxicity to the optic pathways were noted. No patterns of acute and chronic toxicity in relation to various treatment-related variables (surgery, chemotherapy or both) were observed.

\section{Discussion}

$\mathrm{SNc}$ is a rare disease that accounts for $3 \%$ of all head and neck carcinomas (28). As SNcs exhibit particular behaviours, such as chemo- and radioresistance, diagnosis at an advanced stage and lack of association with common risk factors including alcohol and tobacco, they should be considered as separate entities and therefore should not be included in the miscellany of head and neck carcinomas (29). Due to the rarity of these lesions, there is lack of consensus regarding their management, and the majority of data are derived from retrospective analyses. Treatment options vary according to disease extension, and surgery is the preferred therapy in the majority of SNc cases. Recurrence rates widely differ among patients and depend on several factors, including histological type, stage, lymph node metastasis and multimodal treatment strategy $(12,16,30)$.

Historically, the median OS rate for locally advanced SNc ranges from $25-50 \%$ in clinical trials, depending on the aforementioned factors (31). In the present study, a CSS rate of $33.3 \%$ was reported, which is consistent with data in the literature.

Advanced stage at diagnosis is acknowledged to have a strong impact on prognosis and, in particular, on the probability of recurrence (32). In clinical trials, it has been observed that locally advanced diseases, namely those with intracranial extension and/or orbital apex involvement, are characterized by a poor prognosis and shorter disease-free survival, OS and PFS $(12,23)$. This was confirmed by the present study, which demonstrated that patients with stage IV disease had a poorer outcome, in terms of CSS rate (87.5 vs. $57.5 \%$; $\mathrm{P}=0.016)$ and PFS rate $(71.4$ vs. $17.1 \% ; \mathrm{P}=0.001)$ compared with patients with stage III disease.

Patients require accurate selection of treatment in order to maximise response to therapy and survival. For example, there is a wealth of data suggesting that the expression of certain biomarkers, including epidermal growth factor receptor, P16 and survivin, is associated with a more positive response to chemoradiotherapy $(33,34)$.The possibility to pursue a multimodal strategy, namely surgery followed by adjuvant radio or chemoradiotherapy, has been associated with positive outcomes in clinical trials $(35,36)$. The addition of surgical resection did not impact the 2-year CSS rate in the present study, and patients that were treated with upfront surgery followed by adjuvant radio- or chemoradiotherapy did not demonstrate a better survival rate compared with patients treated with exclusive radiotherapy with or without chemotherapy. This may be explained by the fact that surgery was non-radical in the majority of patients, with 7/11 patients undergoing an R1 resection.

SNUc, in addition to intestinal-like and squamous cell histologies, have been reported to correlate with poor prognosis in clinical trials $(37,38)$. Conversely, in the present analysis, histological type did not affect patient prognosis; however, this may be due to the small sample size and the low number of SNUc cases included (3/30).

Finally, the presence of lymph node metastasis, concurrent chemotherapy administration, site of origin and tumour grade did not impact patient prognosis in the current study; however, this may also be due to the small sample size in the framework of a wide heterogeneity.

In the present analysis, acute toxicity was mild and was characterized by mucositis, xerostomia, dysphagia and in-field skin erythema. Grade 3 toxicity was observed in $23.3 \%$ of the patients, while only grade 1 and 2 toxicity were reported in the remaining $76.7 \%$. This toxicity spectrum is considered to be uniquely linked to radiation therapy and, notably, the addition of surgery did not exacerbate it.

Additionally, late toxicity was mild and presented as xerostomia in $13.3 \%$ of patients, while no blindness or optic pathway disruption was reported. This is most likely due the fact that the dose constraints for the OARs considered were not violated in any cases.

In conclusion, the current study was a small retrospective analysis, but did observe a fairly positive survival rate in a group of poor prognosis patients with locally advanced SNc, at the cost of acute and chronic moderate toxicity. Disease stage was the only factor identified to impact prognosis. It is therefore considered that radical surgery should be avoided, given the high rate of recurrence observed in patients, even if they are subsequently treated with adjuvant radio- or chemoradiotherapy.

\section{References}

1. Mahalingappa YB and Khalil HS: Sinonasal malignancy: Presentation and outcomes. J Laryngol Otol 128: 654-657, 2014.

2. Dulguerov P, Jacobsen MS, Allal AS, Lehmann W and Calcaterra T: Nasal and paranasal sinus carcinoma: Are we making progress? A series of 220 patients and a systematic review. Cancer 92: 3012-3029, 2001.

3. Edge S, Bird D and Compton C (eds): AJCC Cancer Staging Manual. 7th edition. New York, Springer, 2010

4. Van Eyken E (ed): Cancer incidence in Flanders, 1997-1999. VLK, Flemish Cancer Network, Brussels, 2002.

5. Périé S, Meyers M, Mazzaschi O, De Crouy Chanel O, Baujat B, Lacau St Guily J. Epidemiology and anatomy of head and neck cancers. Bull Cancer 101: 404-410, 2014 (In French). 
6. Katz TS, Mendenhall WM, Morris CG, Amdur RJ, Hinerman RW and Villaret DB: Malignant tumors of the nasal cavity and paranasal sinuses. Head Neck 24: 821-829, 2002.

7. Khademi B, Moradi A, Hoseini S and Mohammadianpanah M: Malignant neoplasms of the sinonasal tract: Report of 71 patients and literature review and analysis. Oral Maxillofac Surg 13: 191-199, 2009.

8. Dirix P, Nuyts S, Vanstraelen B, Nulens A, Hermans R, Jorissen M, Vander Poorten V and Van den Bogaert W: Post-operative intensity-modulated radiotherapy for malignancies of the nasal cavity and paranasal sinuses. Radiother Oncol 85: 385-391, 2007.

9. Hanna E, DeMonte F, Ibrahim S, Roberts D, Levine N and Kupferman M: Endoscopic resection of sinonasal cancers with and without craniotomy: Oncologic results. Arch Otolaryngol Head Neck Surg 135: 1219-1224, 2009.

10. Blanco AI, Chao KS, Ozyigit G, Adli M, Thorstad WL, Simpson JR, Spector GJ, Haughey B and Perez CA: Carcinoma of paranasal sinuses: Long-term outcomes with radiotherapy. Int J Radiat Oncol Biol Phys 59: 51-58, 2004.

11. Hoffman TK: Systemic therapy strategies for head and neck carcinomas: Current status. GMS Curr Top Otorhinolaryngol Head Neck Surg 11: Doc03, 2012.

12. Kazi M, Awan S, Junaid M, Qadeer S and Hassan NH Management of sinonasal tumors: Prognostic factors and outcomes: A 10 year experience at a tertiary care hospital. Indian J Otolaryngol Head Neck Surg 65 (Suppl 1): S155-S159, 2013.

13. Harvey RJ and Dalgorf DM. Chapter 10: Sinonasal malignancies. Am J Rhinol Allergy 27 (Suppl 1): S35-S38, 2013.

14. Smith SP, Russell JL, Chen NW, Kuo YF and Resto VA: Sinonasal carcinoma: Racial and ethnic disparities in survival-a review of 4,714 patients. Otolaryngol Head Neck Surg 153: 551-560, 2015.

15. Enepekides DJ: Sinonasal undifferentiated carcinoma: An update. Curr Opin Otolaryngol Head Neck Surg 13: 222-225, 2005.

16. Dirix P, Vanstraelen B, Jorissen M, Vander Poorten V and Nuyts S: Intensity-modulated radiotherapy for sinonasal cancer: Improved outcome compared to conventional radiotherapy. Int J Radiat Oncol Biol Phys 78: 998-1004, 2010.

17. Shukovsky LJ and Fletcher GH: Retinal and optic nerve complications in a high dose irradiation technique of ethmoid sinus and nasal cavity. Radiology 104: 629-634, 1972.

18. Ellingwood K and Million R: Cancer of the nasal cavity and ethmoid/sphenoid sinuses. Cancer 43: 1517-1526, 1979.

19. Katz TS, Mendenhall WM, Morris CG, Amdur RJ, Hinerman RW and Villaret DB: Malignant tumors of the nasal cavity and paranasal sinuses. Head Neck 24: 821-829, 2002.

20. Jacobs C, Lyman G, Velez-García E, Sridhar KS, Knight W, Hochster H, Goodnough LT, Mortimer JE, Einhorn LH, Schacter L, et al: A phase III randomized study comparing cisplatin and fluorouracil as single agents and in combination for advanced squamous cell carcinoma of the head and neck. J Clin Oncol 10: 257-263, 1992.

21. Okano S, Tahara M, Zenda S, Fuse N, Yoshino T, Doi T, Kawashima M, Ogino T, Hayashi R and Ohtsu A: Induction chemotherapy with docetaxel, cisplatin and S-1 followed by proton beam therapy concurrent with cisplatin in patients with T4b nasal and sinonasal malignancies. Jpn J Clin Oncol 42: 691-696, 2012

22. Caponigro F, Longo F, Perri F and Ionna F: Docetaxel in the management of head and neck cancer. Anticancer Drugs 20: 639-645, 2009
23. Burtness B, Goldwasser MA, Flood W, Mattar B, Forastiere AA Eastern Cooperative Oncology Group: Phase III randomized trial of cisplatin plus placebo compared with cisplatin plus cetuximab in metastatic/recurrent head and neck cancer: An eastern cooperative oncology group study. J Clin Oncol 23: 8646-8654, 2005

24. NationalComprehensiveCancer Network(NCCN): NCCNClinical Practice Guidelines in Oncology. https://www.ncen.org/professionals/physician gls/f guidelines.asp\#site. Accessed January 21, 2016.

25. Cox JD, Stetz J and Pajak TF: Toxicity criteria of the Radiation Therapy Oncology Group (RTOG) and the European Organization for Research and Treatment of Cancer (EORTC). Int J Radiat Oncol Biol Phys 31: 1341-1346, 1995.

26. National Comprehensive Cancer Network (NCCN): Head and Neck Cancers. Version 1.2015. https://www.nccn.org/professionals/physician_gls/pdf/head-and-neck.pdf. Accessed January 21 , 2016.

27. Sakata K, Maeda A, Rikimaru H, Ono T, Koga N, Takeshige N, Tokutomi T, Umeno H, Kiyokawa K, Morioka M. Advantage of extended craniofacial resection for advanced malignant tumors of the nasal cavity and paranasal sinuses: Long-term outcome and surgical management. World Neurosurg 89: 240-254, 2016.

28. Ansa B, Goodman M, Ward K, Kono SA, Owonikoko TK, Higgins K, Beitler JJ, Grist W, Wadsworth T, El-Deiry M, et al: Paranasal sinus squamous cell carcinoma incidence and survival based on Surveillance, Epidemiology, and End Results data, 1973 to 2009. Cancer 119: 2602-2610, 2013.

29. Lopez F, Suárez V, Vivanco B, Suárez C and Llorente JL: Current management of sinonasal undifferentiated carcinoma. Rhinology 53: 212-220, 2015.

30. Hoppe BS, Nelson CJ, Gomez DR, Stegman LD, Wu AJ, Wolden SL, Pfister DG, Zelefsky MJ, Shah JP, Kraus DH and Lee NY: Unresectable carcinoma of the paranasal sinuses: Outcomes and toxicities. Int J Radiat Oncol Biol Phys 72: 763-769, 2008.

31. Budihna M and Smid L: Carcinoma of the paranasal sinuses: Results of treatment and some prognostic factors. Strahlenther Onkol 168: 322-327, 1992.

32. Bossi P, Saba NF, Vermorken JB, Strojan P, Pala L, de Bree R, Rodrigo JP, Lopez F, Hanna EY, Haigentz M, et al: The role of systemic therapy in the management of sinonasal cancer: A critical review. Cancer Treat Rev 41: 836-843, 2015.

33. Stasikowska-Kanicka O, Wagrowska-Danilewicz $M$ and Danilewicz M: Immunohistochemical study on survivin in sinonasal tumors and its relationship with the immunoexpression of Ki67 and Bcl-2. Folia Histochem Cytobiol 51: 225-231, 2013.

34. Takahashi Y,Bell D, Agarwal G, Roberts D, Xie TX,El-Naggar A, Myers JN and Hanna EY: Comprehensive assessment of prognostic markers for sinonasal squamous cell carcinoma. Head Neck 36: 1094-1102, 2014.

35. Michel J, Fakhry N, Mancini J, Braustein D, Moreddu E, Giovanni A and Dessi P: Sinonasal squamous cell carcinomas: Clinical outcomes and predictive factors. Int J Oral Maxillofac Surg 43: 1-6, 2014

36. Devaraja K, Sikka K, Kumar R and Thakar A: Sinonasal malignancies: Long term follow up after surgical management-an analysis of outcomes. Indian J Otolaryngol Head Neck Surg 67: 28-33, 2015

37. Levine PA, Frierson HF Jr, Stewart FM, Mills SE, Fechner RE and Cantrell RW: Sinonasal undifferentiated carcinoma: A distinctive and highly aggressive neoplasm. Laryngoscope 97: 905-908, 1987.

38. Deutsch BD, Levine PA, Stewart FM, Frierson HF Jr and Cantrell RW: Sinonasal undifferentiated carcinoma: A ray of hope. Otolaryngol Head Neck Surg 108: 697-700, 1993. 